\title{
Memory retrieval before or after extinction reduces recovery of fear in adolescent rats
}

\author{
Kathryn D. Baker, ${ }^{1}$ Gavan P. McNally, and Rick Richardson \\ School of Psychology, The University of New South Wales, Sydney 2052, Australia
}

\begin{abstract}
Adolescent rats exhibit impaired extinction retention compared to pre-adolescent and adult rats. A single nonreinforced exposure to the conditioned stimulus (CS; a retrieval trial) given shortly before extinction has been shown in some circumstances to reduce the recovery of fear after extinction in adult animals. This study investigated whether a retrieval-extinction procedure would reduce the recovery of extinguished fear in adolescent rats. Furthermore, the effect of the retrievalextinction sequence on fear recovery was examined by presenting the retrieval trial following extinction to some animals. In Experiment 1 adolescent rats received one nonreinforced CS presentation (a retrieval trial) or equivalent context exposure (no retrieval) $10 \mathrm{~min}$ before fear extinction. A retrieval trial shortly before extinction reduced overall levels of fear in both test contexts (i.e., it improved extinction retention and reduced renewal). In Experiment 2 a weakening of renewal was observed with a retrieval-extinction manipulation, regardless of whether the retrieval trial occurred in the training or extinction context. A key result was that a retrieval trial $10 \mathrm{~min}$, but not $6 \mathrm{~h}$, after extinction led to reduced overall levels of fear similar to that observed if the retrieval trial was given before extinction (Experiments 3 and 4), inconsistent with the current interpretation of the reduction in relapse being due to a disruption of reconsolidation. Together, these findings show that the impaired extinction retention observed in adolescents can be ameliorated by a very simple behavioral manipulation, but also raise some questions about the mechanisms underlying the retrieval-extinction effect.
\end{abstract}

The extinction of learned fear is a useful animal model for exposure-based therapies that are used to treat anxiety disorders in humans (Myers and Davis 2002). In extinction, repeated presentation of a previously trained conditioned stimulus (CS) in the absence of an aversive unconditioned stimulus (US) reduces fear elicited by the CS. It is thought that extinction involves new learning of a CS-no US association and therefore leads to competing fear and extinction memories. As a consequence, extinguished fear responses are often prone to recovery. For example, extinguished fear responses often spontaneously recover with the passage of time (Quirk 2002; Rescorla 2004) or when the CS is presented in a different physical context to the extinction context, an effect referred to as renewal (Bouton and Bolles 1979a). In addition, conditioned responses which have been extinguished often return following an unexpected aversive event or re-presentation of the US, which is referred to as reinstatement (Bouton and Bolles 1979b).

One approach to reducing the recovery of extinguished fear is based on the notion of disrupting memory reconsolidation. That is, it has been suggested that first reactivating a fear memory and then extinguishing it leads to a disruption of memory reconsolidation and potentially erasure of the original fear memory. In the first demonstration of this, Monfils et al. (2009) reported in adult rats that a single retrieval trial (a nonreinforced exposure to the CS) given shortly before extinction reduced renewal, reinstatement, and spontaneous recovery of learned fear. Schiller et al. (2010) translated this procedure to humans; perhaps the most amazing finding of that study was that humans given a single retrieval trial $10 \mathrm{~min}$ prior to extinction showed no reinstatement of fear even when tested 1 yr later. However, there are several reports where extinction learning after retrieval did not prevent the recovery of fear in adult humans (Golkar et al. 2012; Kindt

\footnotetext{
${ }^{1}$ Corresponding author

E-mail k.baker@unsw.edu.au

Article is online at http://www.learnmem.org/cgi/doi/10.1101/Im.031989.113.
}

and Soeter 2013) and adult mice (Stafford et al. 2013), and even increased the recovery of fear in adult rats (Chan et al. 2010).

Several recent studies have shown that there are important differences in the extinction of fear across development. For example, two recent studies reported that, although adolescent rats displayed identical acquisition of extinction to younger and older rats, they displayed a marked impairment of extinction retention (McCallum et al. 2010; Kim et al. 2011). In adolescence mice and humans also show impaired extinction, both the learning and retention of, demonstrating that a deficit in fear regulation occurs during this period of development across species (Pattwell et al. 2012). Given the high prevalence of anxiety disorders during adolescence (Kessler et al. 2012), understanding how to prevent the recovery of extinguished fear in adolescence could provide valuable insight into therapeutic interventions for anxiety disorders during this stage of development. The pharmacological adjunct D-cycloserine is effective in improving extinction retention in the adolescent rat (McCallum et al. 2010). Considering that the brain undergoes substantial maturation until late adolescence (Andersen 2003; Giedd 2004; Gogtay et al. 2004), nonpharmacological alternatives may be preferred provided similar beneficial effects to pharmacological adjuncts are obtained. However, there is currently little empirical evidence for behavioral manipulations which improve extinction in adolescence and prevent the recovery of extinguished fear. One example of an effective behavioral manipulation is that doubling the amount of extinction training leads to adult-like extinction retention in adolescent rats (McCallum et al. 2010) and adolescent mice (Clem and Huganir 2010). There is also evidence that in adolescent mice a retrieval trial before extensive extinction reduced renewal and spontaneous recovery when animals were tested $1 \mathrm{wk}$ later (Clem and Huganir 2010). Unfortunately, extensive extinction training may not be practical in adolescent humans for a number of reasons (e.g., administering extensive exposure-based therapy would be both time consuming and costly, and also increase the likelihood of patients not completing treatment). The present study 
investigated whether a retrieval-extinction procedure would be effective in reducing the recovery of extinguished fear in adolescent rats given a more typical (i.e., not extensive) extinction training session. The typical interpretation for the finding that a retrieval trial before extinction reduces recovery of fear is that there is a disruption of reconsolidation of the original fear memory (Monfils et al. 2009; Schiller et al. 2010; Flavell et al. 2011). To test this interpretation, the effect of the retrieval-extinction sequence on fear recovery was examined by presenting the retrieval trial following extinction. If this procedure leads to an erasure of the original memory because it allows for disruption of memory reconsolidation, then giving the retrieval trial after extinction should have no effect on fear recovery.

\section{Results}

\section{Experiment 1: A retrieval trial prior to extinction improves extinction retention and reduces renewal in adolescent rats}

Experiment 1 examined whether a retrieval-extinction manipulation would be effective in improving extinction retention and reducing renewal in adolescent rats. Animals were trained to fear a white-noise CS by pairing it three times with a footshock (in context A). The following day, rats received one nonreinforced CS presentation (a retrieval trial) or equivalent context exposure (no retrieval) in a second context (B), before being returned to their home cage for $10 \mathrm{~min}$. Fear was then extinguished via 30 nonreinforced CS presentations (in context B). Animals in the No Retrieval group received an extra CS presentation during the extinction session, in order to equate total number of CS exposures across the two conditions. Rats were tested the following $2 \mathrm{~d}$, once in the extinction context and once in the training context to test for renewal (in a counterbalanced order). Renewal is more difficult to detect in adolescent compared to juvenile or adult rats because the adolescent rats show high levels of fear when tested in either the extinction or training contexts (i.e., they exhibit a marked impairment in extinction retention; McCallum et al. 2010; Kim et al. 2011). Therefore, in the present experiments, differential ABA renewal was inferred from a significant difference in levels of freezing when rats were tested in the training context.

Pre-CS freezing before conditioning, retrieval, extinction, and both tests was low for all experiments (all means $<13.3 \%$, SEMs $<6.9 \%)$. Significant group differences in pre-CS freezing were only detected in Experiment 3 during extinction (see Table 1 for pre-CS means for all sessions across all experiments).

In Experiment 1, fear significantly increased across conditioning trials $\left(F_{(1,22)}=28.56, P<0.001\right)$ (see Fig. $\left.1 \mathrm{~A}\right)$. The No Retrieval and Retrieval groups showed a similar rate of acquisition and overall levels of fear (group main effect $F<1$, group $\times$ trial interaction $F<1$ ). The Retrieval group displayed significantly more freezing during the single CS retrieval trial compared with the pre-CS period $\left(F_{(1,11)}=17.10, P=0.002\right)$. There was evidence of within-session extinction in both the No Retrieval and Retrieval groups (Fig. 1B), with freezing significantly decreasing across extinction blocks $\left(F_{(1,22)}=99.56, P<0.001\right)$. The effect of retrieval was not significant $(F<1)$ nor was the interaction of retrieval and extinction block $\left(F_{(1,22)}=1.07, P=0.311\right)$, confirming that the No Retrieval and Retrieval groups showed a similar rate of extinction and overall levels of fear. That is, a retrieval trial prior to extinction did not affect within-session extinction.

At test (see Fig. 1C), animals in the No Retrieval group exhibited the typical adolescent recovery of fear (i.e., an impairment of extinction retention when tested in the extinction context). Rats exhibited significantly higher levels of freezing in the training
Table 1. Mean ( \pm SEM) percent pre-CS freezing before conditioning, retrieval, extinction, and both tests

\begin{tabular}{llll}
\hline Experiment & \multicolumn{3}{c}{ Group } \\
\hline Experiment 1 & No Retrieval & Retrieval & \\
Conditioning & $0(0)$ & $0.2(0.2)$ & \\
Retrieval & $6.5(3.1)$ & $1.3(0.5)$ & \\
Extinction & $6.3(2.6)$ & $4.4(1.8)$ & \\
Test B & $7.1(2.9)$ & $3.5(1.7)$ & \\
Test A & $6.0(3.4)$ & $1.5(1.1)$ & \\
Experiment 2 & No Retrieval & Retrieval (A) & Retrieval (B) \\
Conditioning & $0(0)$ & $0(0)$ & $0(0)$ \\
Retrieval & $8.4(6.9)$ & $7.9(3.6)$ & $3.4(2.7)$ \\
Extinction & $2.1(1.4)$ & $0.8(0.5)$ & $2.9(1.3)$ \\
Test B & $3.9(1.6)$ & $3.8(1.4)$ & $6.3(3.0)$ \\
Test A & $3.9(2.3)$ & $1.9(1.0)$ & $3.0(2.0)$ \\
Experiment 3 & No Retrieval & Retrieval- & Extinction- \\
& & Extinction & Retrieval \\
Conditioning & $0(0)$ & $0(0)$ & $0.3(0.3)$ \\
Retrieval & $4.7(2.0)$ & $5.8(4.1)$ & $3.3(1.8)$ \\
Extinction & $3.1(1.8)$ & $13.3(2.3)$ & $1.8(1.3)$ \\
Test B & $11.9(4.6)$ & $12.5(4.5)$ & $5.0(1.7)$ \\
Test A & $9.4(3.1)$ & $3.8(1.9)$ & $4.5(3.0)$ \\
Experiment 4 & No Retrieval & Retrieval & Retrieval $6 \mathrm{~h}$ \\
& & 10 min & \\
Conditioning & $0(0)$ & $0(0)$ & $0(0)$ \\
Extinction & $6.3(2.4)$ & $2.1(1.3)$ & $6.8(2.1)$ \\
Retrieval & $3.0(1.5)$ & $2.5(1.3)$ & $11.0(6.5)$ \\
Test B & $6.3(3.5)$ & $4.0(2.2)$ & $7.2(1.9)$ \\
Test A & $1.8(1.3)$ & $3.8(2.0)$ & $10.5(4.5)$ \\
\hline
\end{tabular}

${ }^{a}$ Indicates a significant difference between groups in pre-CS levels of freezing (Retrieval-Extinction compared to No Retrieval $[P=0.002]$ and ExtinctionRetrieval $[P<0.001])$.

context compared to the extinction context $\left(F_{(1,22)}=6.87, P=\right.$ $0.016)$, confirming the occurrence of ABA renewal. Most importantly, rats receiving a retrieval trial prior to extinction had significantly lower levels of fear across the two test contexts $\left(F_{(1,22)}=\right.$ $12.31, P=0.002)$. The retrieval by test context interaction was not significant $(F<1)$. Taken together, these findings demonstrate that a retrieval trial prior to extinction improved extinction retention and reduced renewal in adolescent rats.

\section{Experiment 2: A retrieval trial presented in either the training or extinction context prior to extinction reduces renewal in adolescent rats}

There is some evidence in adult rats that the context in which the retrieval trial is given affects its impact on subsequent extinction training (Chan et al. 2010). Therefore, in Experiment 2 we manipulated the context that the retrieval trial was presented in and assessed whether this influenced the recovery of extinguished fear in adolescent rats. In this experiment, one group received no retrieval (context exposure only in either the training or extinction context) followed by extinction. The retrieval groups received a single retrieval trial in either the training context (group Retrieval A) or the extinction context (group Retrieval B) followed by extinction.

Conditioning proceeded as expected (similar to Fig. 1; data not shown), with fear significantly increasing across conditioning trials $\left(F_{(1,37)}=75.21, P<0.001\right)$. Overall levels of CS-elicited freezing and the rate of acquisition did not differ between the groups (group main effects, $F^{\prime} s<1$; group $\times$ trial interactions, $\left.F^{\prime} \mathrm{s}<1\right)$.

Levels of CS-elicited freezing during the single retrieval trial and across blocks of extinction are shown in Figure 2A. There was significantly more freezing during the single CS retrieval trial compared with the pre-CS period when the retrieval trial occurred 
A

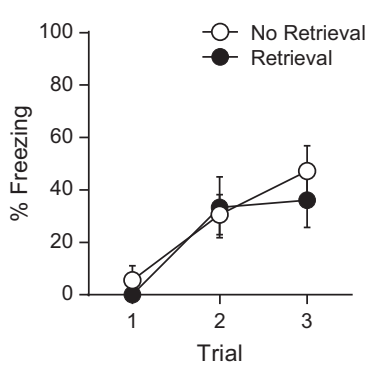

B Retrieval

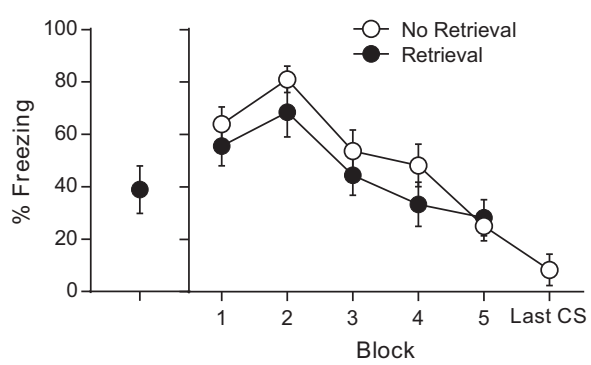

C

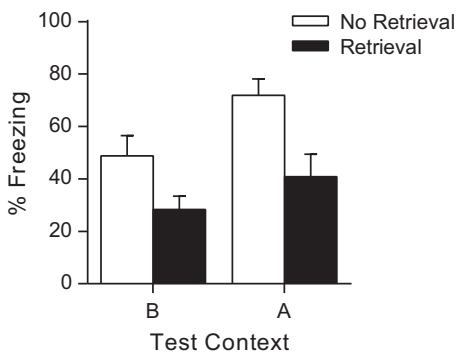

Figure 1. Retrieval-extinction improved extinction retention and reduced renewal. ( $A$ ) Mean ( \pm SEM) levels of CS-elicited freezing during conditioning in Experiment 1 ( $n=12$ for both groups). (B) Mean ( \pm SEM) levels of CS-elicited freezing for retrieval-extinction in Experiment 1. (C) Mean ( \pm SEM) levels of CS-elicited freezing at test in the extinction context B and the training context A in Experiment 1.

in the extinction context $\left(F_{(1,13)}=23.81, P<0.001\right)$ or the training context $\left(F_{(1,11)}=6.32, P=0.016\right)$. During extinction, there was a significant main effect of extinction block $\left(F_{(1,28)}=\right.$ 275.37, $P<0.001)$ but no significant differences between groups (group main effects, largest $F_{(1,37)}=1.48, P=0.231$; group $\times$ extinction block interactions, largest $\left.F_{(1,37)}=1.46, P=0.235\right)$, indicating that there were no differences between groups in the rate of extinction or overall levels of fear.

Test performance is shown in Figure 2B. A single retrieval trial in either the extinction or training context before extinction significantly reduced levels of freezing at test across both contexts $\left(F_{(1,37)}=4.21, P=0.047\right)$. Animals that received the single retrieval trial prior to extinction had similar levels of fear at test across both test contexts, regardless of which context the single retrieval trial was presented in $(F<1)$. There was no significant effect of test context $\left(F_{(1,37)}=2.77, P=0.104\right)$. Although the group (No Retrieval vs. Retrieval groups) by test context interaction was not significant $\left(F_{(1,37)}=1.57, P=0.218\right)$, follow-up tests suggested that retrieval before extinction reduced levels of freezing in the training context (less renewal in these groups compared to the No Retrieval group; $\left.F_{(1,37)}=5.35, P=0.026\right)$, regardless of whether the retrieval cue was presented in the training or extinction context $(F<1)$. However, a retrieval trial prior to extinction did not significantly improve extinction retention in this experiment $\left(F_{(1,37)}=1.58, P=0.217\right)$. That is, giving a single retrieval trial prior to extinction did not affect performance when rats were tested in the extinction context. Therefore, the findings of Experiment 2 partially confirm those of Experiment 1 in that a single retrieval trial in the same context as extinction weakened renewal; the results of this experiment extend that finding by demonstrating that a similar protection against renewal can be gained by giving the single retrieval trial in the training context.

\section{Experiment 3: A retrieval trial after extinction also improves extinction retention and reduces renewal}

The typical interpretation for the finding that a retrieval trial before extinction reduces recovery of fear is that there is a disruption of reconsolidation of the original fear memory (Monfils et al. 2009; Schiller et al. 2010; Flavell et al. 2011). To test this interpretation, we examined the effect of the retrieval-extinction seExperiment 2. quence on fear recovery by presenting the retrieval trial following extinction. If the disrupted reconsolidation perspective is correct, then presenting a retrieval trial after extinction should have no effect on fear recovery because extinction must follow retrieval for the reconsolidation of the fear memory to be disrupted. In Experiment 3, there were three groups: The first didn't receive a retrieval trial before or after extinction, the second group received a single retrieval trial before extinction (as in Experiments 1 and 2), and the third group received a single retrieval trial after extinction. All groups received a retrieval trial or equivalent context exposure in the same context as extinction.

Conditioning proceeded as expected (similar to Fig. 1; data not shown), with fear significantly increasing across conditioning trials $\left(F_{(1,25)}=40.74, P<0.001\right)$. The rate of acquisition and overall levels of fear did not differ between groups (group main effects, largest $F_{(1,25)}=1.64, P=0.212$; group $\times$ trial interactions, largest $\left.F_{(1,25)}=2.16, P=0.155\right)$.

Levels of CS-elicited freezing for the single retrieval trial and across blocks of extinction are shown in Figure 3A. Animals in the Retrieval-Extinction group $\left(F_{(1,9)}=16.18, P=0.003\right)$ and the Extinction-Retrieval group $\left(F_{(1,9)}=15.05, P=0.004\right)$ displayed significantly more freezing during the single CS retrieval trial compared with the pre-CS period. During extinction, statistical analyses revealed that freezing significantly decreased across trials $\left(F_{(1,25)}=175.82, P<0.001\right)$. All groups showed similar withinsession extinction. There was no difference in levels of overall freezing between rats in the Retrieval groups compared to the No Retrieval group $\left(F_{(1,25)}=1.65, P=0.210\right)$, or between those
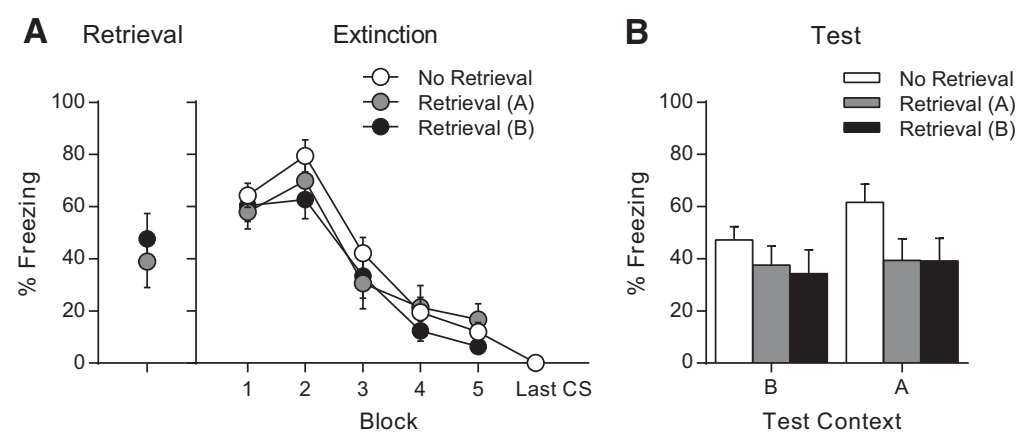

Figure 2. A retrieval trial before extinction reduced renewal regardless of whether the retrieval was presented in the training context (group Retrieval A) or the extinction context (group Retrieval B). Rats were in one of three groups: No Retrieval $(n=14)$, Retrieval (A) $(n=12)$, Retrieval $(B)(n=14)$. $(A)$ Mean $( \pm$ SEM) levels of CS-elicited freezing for retrieval-extinction in Experiment 2. (B) Mean ( \pm SEM) levels of CS-elicited freezing at test in the extinction context $B$ and the training context $A$ in 

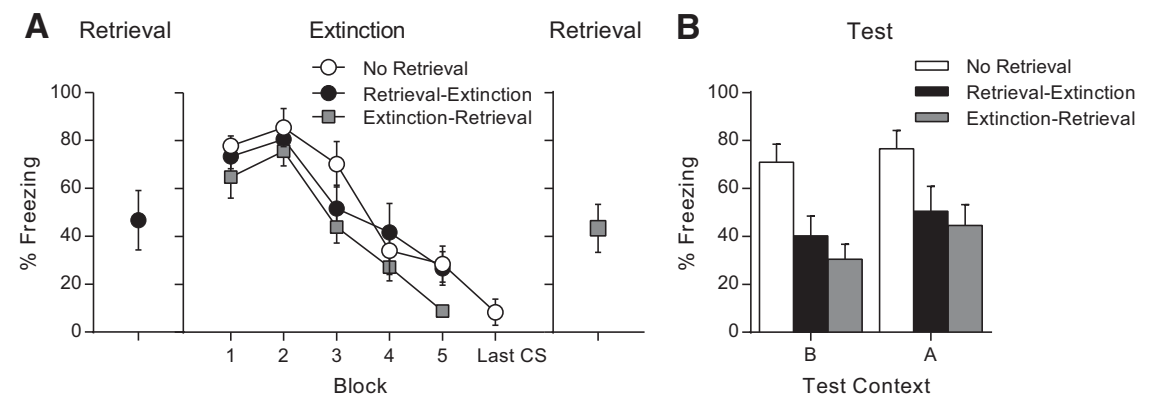

Figure 3. Extinction-retrieval improved extinction retention and reduced renewal. $(A)$ Mean $( \pm$ SEM) levels of CS-elicited freezing for retrieval 10 min before or after extinction in Experiment 3. $(B)$ Mean $( \pm$ SEM) levels of CS-elicited freezing at test in the extinction context $B$ and the training context $\mathrm{A}$ in Experiment 3. Rats were in one of three groups: No Retrieval $(n=8)$, RetrievalExtinction $(n=10)$, Extinction-Retrieval $(n=10)$.

groups given retrieval before or after extinction $\left(F_{(1,25)}=1.75, P=\right.$ $0.198)$. The rate of extinction was also similar across groups (Retrieval groups vs. No Retrieval, $F<1$; retrieval before or after extinction, $\left.F_{(1,25)}=1.14, P=0.295\right)$.

At test (see Fig. 3B), animals in the retrieval groups showed lower levels of freezing across both test contexts compared to the rats in the No Retrieval group $\left(F_{(1,25)}=13.23, P<0.001\right)$; retrieval before or after extinction resulted in similar levels of overall freezing $(F<1)$. The effect of test context was not significant $\left(F_{(1,25)}=3.46, P=0.075\right)$ nor were the interactions of retrieval group with test context $\left(F^{\prime} \mathrm{s}<1\right)$. This shows that a retrieval trial either before or after extinction improved extinction retention in adolescent rats and also reduced renewal. That is, a single retrieval trial following extinction produced the same protection against recovery of fear as a single retrieval trial before extinction.

\section{Experiment 4: A retrieval trial after extinction is more effective in reducing the recovery of fear when presented shortly after extinction than when delayed}

Experiment 3 found that a single retrieval trial following extinction was as effective in enhancing extinction retention and reducing renewal in adolescent rats as was giving such a trial before extinction training. This result was somewhat surprising, and does not fit in with the disrupted reconsolidation idea normally offered to account for the retrieval-extinction effect. Therefore, Experiment 4 was designed to replicate that finding as well as determine the effect of delaying the single retrieval trial following extinction. A prior study demonstrated that a retrieval trial before extinction was more effective in reducing spontaneous recovery when presented $10 \mathrm{~min}$ or $1 \mathrm{~h}$ before extinction than when it occurred $6 \mathrm{~h}$ before extinction (Monfils et al. 2009). This effect has been interpreted as demonstrating the temporal boundary conditions for the effectiveness of retrieval-extinction, or a reconsolidationblockade window. Therefore, in the present experiment one group only received the extinction training (context exposure only either $10 \mathrm{~min}$ or $6 \mathrm{~h}$ after extinction), another group received a single retrieval trial 10 min after extinction, and a third group received a single retrieval trial $6 \mathrm{~h}$ after extinction.

Conditioning proceeded as expected (similar to Fig. 1; data not shown), with fear significantly increasing across
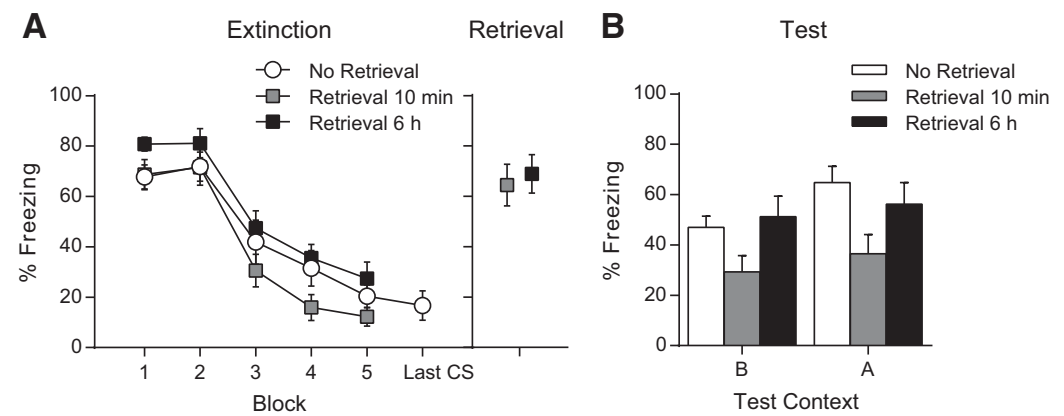

Figure 4. A retrieval trial after extinction is more effective in reducing fear recovery when presented shortly after extinction than when delayed. $(A)$ Mean $( \pm$ SEM) levels of CS-elicited freezing for retrieval either $10 \mathrm{~min}$ or $6 \mathrm{~h}$ after extinction in Experiment 4. (B) Mean ( \pm SEM) levels of CS-elicited freezing at test in the extinction context B and the training context A in Experiment 4 ( $n=15$ for each group). conditioning trials $\left(F_{(1,42)}=70.22, P<\right.$ 0.001). All groups showed similar levels of CS-elicited freezing during conditioning and a similar rate of acquisition (group main effects, largest $F_{(1,42)}=$ $1.83, P=0.183$; group $\times$ trial interactions, largest $\left.F_{(1,42)}=1.09, P=0.303\right)$. There was evidence of within-session extinction across all groups (Fig. 4A), with freezing significantly decreasing across extinction blocks $\left(F_{(1,42)}=260.04, P<\right.$ $0.001)$. Although animals in the No Retrieval and Retrieval 6-h groups exhibited similar levels of freezing during extinction $\left(F_{(1,42)}=1.45, P=0.235\right)$, the rats which received a retrieval trial $10 \mathrm{~min}$ after extinction exhibited a trend toward lower levels of freezing during extinction $\left(F_{(1,42)}=3.71, P=0.061\right)$, but this was not significant. There were no significant interactions of retrieval group with extinction block (largest $F_{(1,42)}=1.55, P=0.220$ ), indicating that all groups showed a similar rate of extinction. Animals given a single retrieval trial either $10 \mathrm{~min}$ or $6 \mathrm{~h}$ after extinction exhibited significantly more freezing (smallest $F_{(1,14)}=25.73, P<0.001$ ) during the single CS retrieval trial than during the pre-CS period (Fig. $4 \mathrm{~A}$ ).

Test performance is shown in Figure 4B. Rats showed a trend toward higher levels of freezing in the training context (ABA renewal) than in the extinction context $\left(F_{(1,42)}=3.98, P=0.053\right)$. Although animals in the No Retrieval and Retrieval 6-h groups exhibited similar levels of overall freezing across the two test contexts $(F<1)$, the rats that received a single retrieval trial $10 \mathrm{~min}$ after extinction showed significantly lower overall levels of freezing across both test contexts $\left(F_{(1,42)}=10.08, P=0.003\right)$. The interactions between group and test context were not significant (largest $F_{(1,42)}=$ $1.09, P=0.302)$. These findings replicate the results of Experiment 3 and further show that a single retrieval trial is more effective in reducing recovery of fear and renewal when presented shortly after extinction than when given after a 6-h delay.

\section{Discussion}

Adolescent animals exhibit impaired fear extinction retention McCallum et al. 2010; Kim et al. 2011; Pattwell et al. 2012), but in the present study we have demonstrated a simple behavioral manipulation which prevents this impairment. Specifically, although the retrieval-extinction procedure did not significantly affect levels of fear during extinction, it did result in significantly less fear at test, in both the extinction and fear conditioning 
contexts. Previous findings in adult rats have demonstrated that before extinction, a retrieval trial in the training context, but not the extinction context, deepened extinction and produced a more rapid re-extinction of renewed fear (Chan et al. 2010). In adolescent rats we found that the retrieval-extinction manipulation reliably weakened renewal, regardless of whether the retrieval trial occurred in the training or extinction context. We also demonstrated that a single retrieval trial following extinction was as effective in enhancing extinction retention and reducing renewal as was giving such a trial before extinction training. These findings compliment a recent study that showed giving adolescent rats double the amount of extinction training improved extinction retention (Kim et al. 2011). In addition, when adolescent mice are given a retrieval trial before extensive extinction training sufficient to obtain adult-like extinction retention, the retrieval-extinction manipulation reduced renewal and spontaneous recovery (Clem and Huganir 2010). Importantly, in the current study only a single retrieval trial, rather than 19-30 additional extinction trials, was found to improve extinction retention as well as reduce renewal in adolescent rats. The findings reported in this study also support previous demonstrations in adult rats that a retrieval-extinction procedure reduces renewal of fear (Monfils et al. 2009; Flavell et al. 2011) and several forms of reinstatement of drug seeking (Ma et al. 2012; Xue et al. 2012; Millan et al. 2013). However, several other studies have found that extinction learning after a retrieval trial did not prevent the recovery of fear in adult humans (Golkar et al. 2012; Kindt and Soeter 2013) and even increased the recovery of fear in adult rats (Chan et al. 2010). Further research examining the mechanisms underlying how retrieval-extinction manipulations produce a loss of fear will be useful for understanding the discrepancies between these findings.

It has been suggested that once reactivated, a consolidated memory enters a labile state which is susceptible to disruption (Nader et al. 2000; Parsons et al. 2006). The process where memories are restabilized following reactivation is referred to as "reconsolidation" (Lee 2008). The reported loss of fear produced by the retrieval-extinction manipulation is typically interpreted as a disruption of reconsolidation (Monfils et al. 2009; Schiller et al. 2010; Flavell et al. 2011). From this perspective, when extinction follows memory reactivation it leads to some erasure of the original memory. In contrast, unlearning, or erasure, of the original memory is not typically thought to occur with normal extinction. Instead, extinction is thought to result in new learning which actively inhibits the original memory (Myers and Davis 2002; Quirk and Mueller 2008). One interpretation of the current results is that the retrieval-extinction procedure led to some erasure of the original fear memory in the adolescent rats and therefore caused the improved extinction retention and reduced renewal of extinguished fear.

If this is, indeed, the mechanism responsible, then a likely locus for the weakening of the fear memory trace is the amygdala. In support of this, retrieval-extinction manipulations have been found to reduce activity in the amygdala in humans (Agren et al. 2012). In addition, multiple, closely timed retrievals of conditioned fear produce molecular changes to the phosphorylation state of synaptic GluR1 receptors in the amygdala (Jarome et al. 2012) at a site (Ser845) which controls the stability of calciumpermeable GluR2-lacking AMPA receptors and therefore may lead to AMPA receptor endocytosis (Ehlers 2000). Importantly, dephosphorylation of GluR1 at Ser845 occurs in the amygdala when two CSs are presented 1-h apart, but not when they are presented either 3-min or 24-h apart (Monfils et al. 2009; Jarome et al. 2012). Given that having an interval of either $10 \mathrm{~min}$ or $1 \mathrm{~h}$ between a retrieval trial and extinction effectively reduces the recovery of extinguished fear it is possible that a dephosphorylation of GluR1 occurs in the amygdala when extinction closely follows memory retrieval (Monfils et al. 2009). Related to this, Clem and Huganir (2010) demonstrated that the retrieval-extinction procedure resulted in a loss of calcium-permeable AMPA receptors and consequently a reduction in synaptic transmission in the lateral amygdala. Therefore, extinction after a recent, but not too recent, retrieval event may facilitate the removal of AMPA receptors from the synapse via dephosphorylation of GluR1. These molecular changes would be consistent with a reversal of conditioning-induced molecular changes in the amygdala.

However, the difficulty with this account is that it requires retrieval to precede extinction in order to produce long-lasting decrements in fear. A key result from this study was that the order of retrieval and extinction sessions was irrelevant to the behavioral outcome. Retrieval after, or prior, to extinction yielded greater long-term decrements in fear. This finding that retrieval after extinction reduced the recovery of extinguished fear is inconsistent with a disruption of reconsolidation account because it is difficult to explain how the original memory trace would be destabilized when retrieval follows extinction. However, it is consistent with a recent demonstration that reversal of retrieval and extinction sessions produced the same protection against context-induced reinstatement (i.e., ABA renewal) of alcoholic beer seeking in adult rats as did retrieval before extinction (Millan et al. 2013). Therefore, it would have to be the case that either (1) different mechanisms underlie the reduction in fear following these two procedures or (2) if the same mechanism underlies these two procedures, then that mechanism is not likely to be a disruption of memory reconsolidation.

One idea that fits with the second alternative is that retrievalextinction may augment or deepen extinction (Xue et al. 2012), rendering extinguished fear more resistant to recovery. This explanation can account for both retrieval-extinction and extinction-retrieval improving extinction retention and attenuating renewal. The common mechanism here would be the spacing of extinction trials. There is some evidence that spaced extinction trials reduces the recovery of fear (e.g., renewal) compared to massed extinction trials (Urcelay et al. 2009). Another study, using contextual fear conditioning, suggests that spaced extinction trials, while less effective in reducing short-term conditioned responding (i.e., across extinction trials), produce better long-term response loss (when animals are tested $24 \mathrm{~h}$ after extinction; $\mathrm{Li}$ and Westbrook 2008). This may be particularly beneficial for adolescent rats given that they exhibit impaired long-term extinction retention. However, increased spacing between extinction trials with retrieval-extinction and extinction-retrieval cannot be the only contributing factor driving decrements in conditioned fear with these procedures because a retrieval trial either $6 \mathrm{~h}$ before extinction (Monfils et al. 2009) or $6 \mathrm{~h}$ after extinction (Experiment 4) was less effective than retrieval $10 \mathrm{~min}$ before or after extinction. To explain why the interval between extinction sessions would influence the effectiveness of retrieval-extinction procedures, Xue et al. (2012) suggested that intermittent exposure to extinction training during the consolidation period of a retrieval session may strengthen the extinction memory, but exposure outside the consolidation window (i.e., $6 \mathrm{~h}$ ) would be ineffective.

The results reported in this study show that a retrieval trial before or after extinction reliably augments the loss of fear in adolescent rats. In two out of four experiments we observed higher levels of fear in a context different than that used for extinction training (i.e., we observed the renewal effect). The most likely reason for why this effect was not observed in the other two experiments is that adolescent rats exhibit impaired extinction recall, leading to high levels of fear even when tested in the extinction context the next day (McCallum et al. 2010; Kim et al. 2011). The retrieval-extinction manipulations have two benefits as they overcome the impairment of extinction retention typically 
observed in adolescent rats as well as prevent another form of recovery of fear (i.e., renewal). These findings may help to inform treatments for anxiety in adolescence by suggesting a nonpharmacological manipulation that can reduce the recovery of extinguished fear. Further research should investigate whether retrieval-extinction manipulations effectively augment the loss of fear in adolescent humans who also show impaired extinction (Pattwell et al. 2012). Adolescents might be especially likely to exhibit beneficial effects of the retrieval-extinction procedure because there are differences in the neural circuitry recruited during fear extinction in adolescent and adult rats. The consolidation of extinction is thought to involve the infralimbic (IL) region of the medial prefrontal cortex (Sotres-Bayon et al. 2009). Compared to adults or juvenile rats which display good extinction retention, the impaired extinction retention in adolescent animals is associated with reduced levels of phosphorylated mitogen-activated protein kinase (pMAPK) in the IL after extinction (Kim et al. 2011). It would be interesting to determine whether both retrieval-extinction and extinction-retrieval recruit the IL to augment extinction retention in adolescent animals. There are some recent findings that are in broad support of this idea. That is, Xue et al. (2012) reported that extinction of cocaine seeking in adult rats increased $\mathrm{PKM} \zeta$ expression in the IL and decreased PKM $\zeta$ expression in the basolateral amygdala, and, importantly, that the retrieval-extinction procedure enhanced both of these extinction-induced changes.

In contrast to these possibilities, retrieval-extinction manipulations may not affect either the original training (through reconsolidation) or extinction memories (through deepened extinction). Instead, these procedures may promote better discrimination at test between the competing training and extinction memories (Millan et al. 2013). In support of this possibility, responses during extinction are not reliably affected by retrieval before extinction, suggesting that extinction may not be deepened. The absence of the shock US following the retrieval trial may set up an expectation of the contingencies present during the later extinction session. In a similar way this applies to the extinction-retrieval manipulation where extinction signals another extinction trial. This enables better discrimination of the training and extinction memories (Redish et al. 2007). Animals which received retrieval-extinction manipulations may be better at adapting to the conditions (i.e., no US) present at both the extinction retention and renewal tests and therefore show reduced fear responses in both test contexts.

In conclusion, we have demonstrated a simple behavioral procedure which prevents the typical adolescent recovery of extinguished fear. A retrieval trial before or after extinction reduced the recovery of extinguished fear in adolescent rats, improving extinction retention and attenuating renewal. The loss of fear was independent of the order of retrieval and extinction trials, inconsistent with a disruption of reconsolidation interpretation. Instead, retrieval around the time of extinction may promote better discrimination of the training and extinction memories. Despite raising potential questions concerning the mechanism underlying the reduction of fear relapse following the retrievalextinction procedure, the present findings suggest that giving a single retrieval trial around the time of extinction training may offer a simple, effective way of enhancing long-term treatment gains for human populations when extinction deficits are seen.

\section{Materials and Methods}

\section{Subjects}

Subjects were experimentally naive adolescent male SpragueDawley rats obtained from the breeding colony maintained by the School of Psychology at The University of New South Wales. Rats were weaned at postnatal day 21-24 and were housed in groups of 6-8 in plastic boxes with food and water available ad libitum. The colony room was maintained on a 12-h light-dark cycle (lights on at 7 a.m.). All procedures were approved by the Animal Care and Ethics Committee at The University of New South Wales and conducted in accordance with the guidelines of the Australian Code of Practice for the Care and Use of Animals for Scientific Purposes (7th ed., 2004). For all experiments, rats were 34-37 d of age at the time of fear extinction.

\section{Apparatus}

Experiments were conducted in two sets of Med Associates chambers $(24-\mathrm{cm}$ length $\times 30$-cm width $\times 21$ - $\mathrm{cm}$ height) to provide two different contexts. All chambers were enclosed within a sound- and light-attenuating cabinet, with constant low-level background noise $(\sim 58 \mathrm{~dB})$ produced by a ventilation fan. Rats were observed using a mounted infrared camera on the rear cabinet wall connected to a DVD recorder and monitor. The chambers were cleaned with tap water after each experimental session.

Rats received conditioning in chambers constructed of a clear Perspex ceiling, rear wall, and hinged front door with stainless-steel side walls (referred to as context A). A stainless-steel tray of bedding was underneath a floor consisting of stainless-steel rods (4-mm diameter, spaced $16 \mathrm{~mm}$ apart center to center). The right side wall contained a speaker located in the top-left corner and a recessed magazine positioned behind a $5 \times 5 \mathrm{~cm}$ opening in the center. Two nose poke holes and two key lights were symmetrically located on the same side wall and were inactive during all experiments. Infrared lighting was the only source of illumination in context $\mathrm{A}$.

Extinction was conducted in a second set of chambers (referred to as context B), which had different visual features, flooring, and lighting to context A. The ceiling and front wall of the context B chambers had paper with vertical black-and-white stripes (2.5-cm width) attached. A clear Perspex insert covered the grid floor and the stainless-steel tray under the floor did not contain any bedding. A white light on top of the chamber provided illumination in context $\mathrm{B}$.

The CS was a white noise; noise levels increased by $4 \mathrm{~dB}$ above background when the CS was presented. The US was a scrambled 0.6-mA, 1-sec shock delivered through the grid floor. A computer running Med-PC IV software (Med Associates) controlled all presentations of the CS and US.

\section{Procedure}

Handling and context preexposure

Rats were handled for 3-4 min for two consecutive days before conditioning to habituate to the experimenter. On each day, following handling animals were placed in the conditioning chamber for $10 \mathrm{~min}$.

\section{Conditioning}

Fear conditioning occurred in context A. After a 2-min adaptation period, rats received three CS-US pairings; each white-noise CS was presented for $10 \mathrm{sec}$ and co-terminated with the shock US. The intertrial intervals (ITIs) were 135 and $85 \mathrm{sec}$ (mean of $110 \mathrm{sec})$.

\section{Retrieval}

Retrieval occurred in the same context as extinction (context B), with the exception of Experiment 2 in which retrieval was presented in either context A or B. After a 2-min adaptation period, rats received one nonreinforced CS presentation (a retrieval trial, $10 \mathrm{sec}$ ) or equivalent context exposure (no retrieval) before being returned to their home cage for 10 min before extinction. In Experiments 3 and 4, the single retrieval trial occurred either 10 min or $6 \mathrm{~h}$ after extinction. 


\section{Extinction}

Extinction occurred in context $\mathrm{B}, \sim 24 \mathrm{~h}$ after conditioning, and consisted of 30 nonreinforced presentations of the CS $(10 \mathrm{sec}$ each, 10-sec ITI, following a 2-min adaptation period). Animals in the No Retrieval group received 31 CS presentations during the extinction session. For statistical analyses of within-session extinction, six extinction trials were averaged to represent one block of extinction.

\section{Test}

Animals were tested on each of the $2 \mathrm{~d}$ after extinction, once in the extinction context (B) and once in the conditioning context (A) to test for renewal. Two minutes after being placed in the chamber, a single CS was presented for 2 min. Test order was counterbalanced. The conditioning, extinction, and test procedures were based upon those previously used in our laboratory for developmental studies of extinction (e.g., McCallum et al. 2010).

\section{Scoring, exclusion criteria, and statistics}

Behavior was scored using a time-sampling procedure every $3 \mathrm{sec}$ as either freezing or not freezing. Freezing was defined as the absence of all movement except that required for breathing (Fanselow 1980). The total observations scored as freezing were summed and converted to a percentage. A second observer unaware of the experimental condition of each rat scored a 30\% sample of test data. The inter-rater reliability was very high $(r=0.96)$. One rat was excluded from analysis in Experiment 4 due to high pre-CS freezing (55\%) in the extinction context at test.

Pre-CS freezing was analyzed using independent measures $t$-tests or ANOVA with Tukey's HSD post hoc test where appropriate. Conditioning, retrieval, extinction, and test data were analyzed using ANOVA with orthogonal contrasts. Type I error rate $(\alpha)$ was controlled at 0.05 for each contrast tested. ABA renewal is somewhat difficult to detect in adolescent rats because they show high levels of fear when tested in either the extinction or training contexts (i.e., they exhibit a marked impairment in extinction retention; McCallum et al. 2010; Kim et al. 2011). Therefore, differential ABA renewal was inferred by a significant difference in levels of freezing when rats were tested in the training context.

\section{Acknowledgments}

This research was supported by the grant DP110100754 from the Australian Research Council to G.P.M. and R.R. K.D.B. is a National Health and Medical Research Council Peter Doherty Early Career Fellow (APP1054642).

\section{References}

Agren T, Engman J, Frick A, Björkstrand J, Larsson E-M, Furmark T, Fredrikson M. 2012. Disruption of reconsolidation erases a fear memory trace in the human amygdala. Science 337: 1550-1552.

Andersen SL. 2003. Trajectories of brain development: Point of vulnerability or window of opportunity? Neurosci Biobehav Rev 27: 3-18.

Bouton ME, Bolles RC. 1979a. Contextual control of the extinction of conditioned fear. Learn Motiv 10: 445-466.

Bouton ME, Bolles RC. 1979b. Role of conditioned contextual stimuli in reinstatement of extinguished fear. J Exp Psychol Anim Behav Process 5: $368-378$.

Chan WY, Leung HT, Westbrook RF, McNally GP. 2010. Effects of recent exposure to a conditioned stimulus on extinction of Pavlovian fear conditioning. Learn Mem 17: 512-521.

Clem RL, Huganir RL. 2010. Calcium-permeable AMPA receptor dynamics mediate fear memory erasure. Science 330: 1108-1112.

Ehlers MD. 2000. Reinsertion or degradation of AMPA receptors determined by activity-dependent endocytic sorting. Neuron 28: $511-525$.

Fanselow M. 1980. Conditional and unconditional components of postshock freezing. Pavlov J Biol Sci 15: 177-182.

Flavell CR, Barber DJ, Lee JLC. 2011. Behavioural memory reconsolidation of food and fear memories. Nat Commun 2: 504.
Giedd JN. 2004. Structural magnetic resonance imaging of the adolescent brain. Ann N Y Acad Sci 1021: 77-85.

Gogtay N, Giedd JN, Lusk L, Hayashi KM, Greenstein D, Vaituzis AC, Nugent TF, Herman DH, Clasen LS, Toga AW, et al. 2004. Dynamic mapping of human cortical development during childhood through early adulthood. Proc Natl Acad Sci 101: 8174-8179.

Golkar A, Bellander M, Olsson A, Öhman A. 2012. Are fear memories erasable?-reconsolidation of learned fear with fear relevant and fear-irrelevant stimuli. Front Behav Neurosci 6: 80.

Jarome TJ, Kwapis JL, Werner CT, Parsons RG, Gafford GM, Helmstetter FJ. 2012. The timing of multiple retrieval events can alter GluR1 phosphorylation and the requirement for protein synthesis in fear memory reconsolidation. Learn Mem 19: 300-306.

Kessler RC, Avenevoli S, Costello EJ, Georgiades K, Green JG, Gruber MJ, He J-p, Koretz D, McLaughlin KA, Petukhova M, et al. 2012. Prevalence, persistence, and sociodemographic correlates of DSM-IV disorders in the National Comorbidity Survey Replication Adolescent Supplement. Arch Gen Psychiatry 69: 372-380.

Kim JH, Li S, Richardson R. 2011. Immunohistochemical analyses of long-term extinction of conditioned fear in adolescent rats. Cereb Cortex 21: 530-538.

Kindt M, Soeter M. 2013. Reconsolidation in a human fear conditioning study: A test of extinction as updating mechanism. Biol Psychol 92: 43-50.

Lee JLC. 2008. Memory reconsolidation mediates the strengthening of memories by additional learning. Nat Neurosci 11: 1264-1266.

Li SH, Westbrook FR. 2008. Massed extinction trials produce better shortterm but worse long-term loss of context conditioned fear responses than spaced trials. J Exp Psychol Anim Behav Process 34: 336-351.

Ma X, Zhang J-J, Yu L-C. 2012. Post-retrieval extinction training enhances or hinders the extinction of morphine-induced conditioned place preference in rats dependent on the retrieval-extinction interval. Psychopharmacology 221: 19-26.

McCallum J, Kim JH, Richardson R. 2010. Impaired extinction retention in adolescent rats: Effects of D-cycloserine. Neuropsychopharmacology 35: 2134-2142.

Millan EZ, Milligan-Saville J, McNally GP. 2013. Memory retrieval, extinction, and reinstatement of alcohol seeking. Neurobiol Learn Mem 101: $26-32$.

Monfils MH, Cowansage KK, Klann E, LeDoux JE. 2009. Extinctionreconsolidation boundaries: Key to persistent attenuation of fear memories. Science 324: 951-955.

Myers KM, Davis M. 2002. Behavioral and neural analysis of extinction. Neuron 36: 567-584.

Nader K, Schafe GE, Le Doux JE. 2000. Fear memories require protein synthesis in the amygdala for reconsolidation after retrieval. Nature 406: $722-726$.

Parsons RG, Gafford GM, Baruch DE, Riedner BA, Helmstetter FJ. 2006. Long-term stability of fear memory depends on the synthesis of protein but not mRNA in the amygdala. Eur J Neurosci 23: 1853-1859.

Pattwell SS, Duhoux S, Hartley CA, Johnson DC, Jing D, Elliott MD, Ruberry EJ, Powers A, Mehta N, Yang RR, et al. 2012. Altered fear learning across development in both mouse and human. Proc Natl Acad Sci 109: 16318-16323.

Quirk GJ. 2002. Memory for extinction of conditioned fear is long-lasting and persists following spontaneous recovery. Learn Mem 9: 402-407.

Quirk GJ, Mueller D. 2008. Neural mechanisms of extinction learning and retrieval. Neuropsychopharmacology 33: 56-72.

Redish A, Jensen S, Johnson A, Kurth-Nelson Z. 2007. Reconciling reinforcement learning models with behavioral extinction and renewal: Implications for addiction, relapse, and problem gambling. Psychol Rev 114: 784-805.

Rescorla RA. 2004. Spontaneous recovery. Learn Mem 11: 501-509.

Schiller D, Monfils MH, Raio CM, Johnson DC, LeDoux JE, Phelps EA. 2010. Preventing the return of fear in humans using reconsolidation update mechanisms. Nature 463: 49-53.

Sotres-Bayon F, Diaz-Mataix L, Bush DEA, LeDoux JE. 2009. Dissociable roles for the ventromedial prefrontal cortex and amygdala in fear extinction: NR2B contribution. Cereb Cortex 19: 474-482.

Stafford JM, Maughan DK, Ilioi EC, Lattal KM. 2013. Exposure to a fearful context during periods of memory plasticity impairs extinction via hyperactivation of frontal-amygdalar circuits. Learn Mem 20: 156-163.

Urcelay GP, Wheeler DS, Miller RR. 2009. Spacing extinction trials alleviates renewal and spontaneous recovery. Learn Behav 37: 60-73.

Xue Y-X, Luo Y-X, Wu P, Shi H-S, Xue L-F, Chen C, Zhu W-L, Ding Z-B, Bao Y-p, Shi J, et al. 2012. A memory retrieval-extinction procedure to prevent drug craving and relapse. Science 336: 241-245.

Received June 3, 2013; accepted in revised form June 20, 2013. 


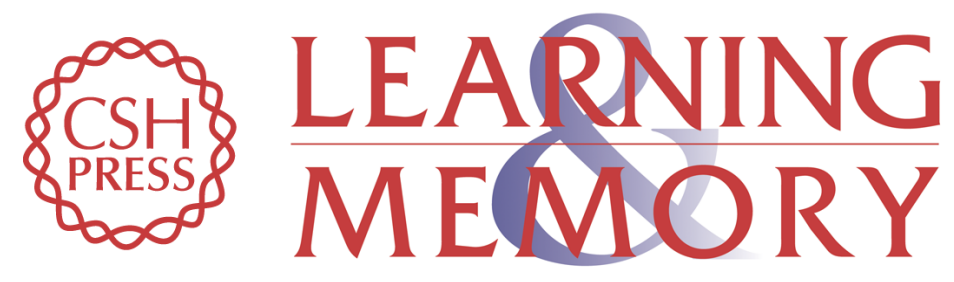

\section{Memory retrieval before or after extinction reduces recovery of fear in adolescent rats}

Kathryn D. Baker, Gavan P. McNally and Rick Richardson

Learn. Mem. 2013, 20:

Access the most recent version at doi:10.1101/Im.031989.113

References This article cites 35 articles, 11 of which can be accessed free at: http://learnmem.cshlp.org/content/20/9/467.full.html\#ref-list-1

Creative This article is distributed exclusively by Cold Spring Harbor Laboratory Press for the Commons License first 12 months after the full-issue publication date (see

http://learnmem.cshlp.org/site/misc/terms.xhtml). After 12 months, it is available under a Creative Commons License (Attribution-NonCommercial 3.0 Unported), as described at http://creativecommons.org/licenses/by-nc/3.0/.

Email Alerting Receive free email alerts when new articles cite this article - sign up in the box at the Service top right corner of the article or click here. 